\title{
Thermal Properties of a Particle Confined to a Parabolic Quantum Well in 2D Space with Conical Disclination
}

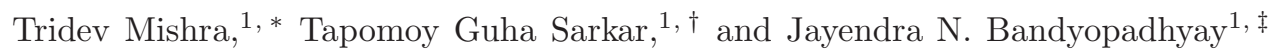 \\ ${ }^{1}$ Department of Physics, Birla Institute of Technology and Science, Pilani 333031, India.
}

\begin{abstract}
The thermal properties of a system, comprising of a spinless non-interacting charged particle in the presence of a constant external magnetic field and confined in a parabolic quantum well are studied. The focus has been on the effects of a topological defect, of the form of conical disclination, with regard to the thermodynamic properties of the system. We have obtained the modifications to the traditional Landau-Fock-Darwin spectrum in the presence of conical disclination. The effect of the conical kink on the degeneracy structure of the energy levels is investigated. The canonical formalism is used to compute various thermodynamic variables. The study shows an interplay between magnetic field, temperature and the degree of conicity by setting two scales for temperature corresponding to the frequency of the confining potential and the cyclotron frequency of external magnetic field. The kink parameter is found to affect the quantitative behaviour of the thermodynamic quantities. It plays a crucial role in the competition between the external magnetic field and temperature in fixing the values of the thermal response functions. This study provides an important motivation for studying similar systems, however with non trivial interactions in the presence of topological defects.
\end{abstract}

PACS numbers: 68.65.Hb, 61.72.Lk, 05.70. -a

\section{INTRODUCTION}

In recent decades, advances in nanotechnology, semiconductor device fabrication and micro-fabrication techniques have thrown open the rich field of two dimensional electron systems (2DES) [1-3]. There is a special focus on systems with confinement along all three spatial dimensions [4 7]. Several experiments aimed at understanding the electronic [2] and optical properties [8, 9] of such systems, commonly referred to as quantum dot [4, 10], have been undertaken [6, 11, 12]. In some of these experiments the quantum dots are exposed to magnetic fields of varying strength and their response is studied in terms of electron transport and inter-band tunneling properties [6, 12 14].

A reasonable model to describe such non-relativistic quantum dot systems requires a parabolic quantum well as the confining potential [15]. However, theoretical exploration of such models is far from exhaustive and presents several potential situations for study. One such aspect is the response of a charged particle confined to a quantum well in an applied magnetic field [16] and constrained to a surface with non-trivial geometry. An often studied topological defect is a conical disclination [17 21], which has been the focus of quantum mechanical problems in curved space [21 24] of the Landau level type [25]. Another dimension of investigation [15, 26] looks into the thermodynamic properties of confined systems of the Landau-Fock-Darwin 27] type in ordinary Euclidean space. These lines of examination can be brought to converge on the issue of thermodynamic behavior of single

\footnotetext{
* tridev.mishra@pilani.bits-pilani.ac.in

$\dagger$ tapomoy1@gmail.com

$\ddagger$ jnbandyo@gmail.com
}

electron confined in the presence of a conical disclination, a situation which has the potency to reveal the physics of quantum dots with novel geometry. The presence of topological defects in the constraining surface is expected to affect the thermodynamic characteristics of such a system and their asymptotic behavior.

In this paper we analyze the properties exhibited by a charged particle constrained on a surface with a defect of the nature of conical disclination. The system comprises of the particle subjected to a magnetic field, while it is trapped in a parabolic Fock-Darwin potential. The approach is, to first calculate the energy spectrum and then use the canonical partition function to uncover the thermodynamic properties of the system. We have used the Schrödinger equation to obtain the energy eigenspectrum. This is motivated by the fact that spectroscopic studies of electronic states of quantum dots (such as InSb quantum dot) indicate that a Schrödinger Hamiltonian with a Fock-Darwin confining potential gives reasonable agreement with experiments [6]. We introduce a conical disclination defect in such systems through the Volterra process [19] (discussed in the next section). The approach here borrows an idea from gravity, whereby the defect appears as a modification of geometry of the underlying space. We also note that a similar approach maybe adopted for graphene [28] like 2D systems. However, the spectrum there is linear at the band minima and thereby the Dirac Hamiltonian has to be adopted. We have studied the variations of the thermodynamic quantities of interest like internal energy, specific heat and entropy with magnetic field, temperature and extent of the defect. The asymptotic limits of these are checked for confirmation with expected results.

The paper consists of four sections. Sec. II is dedicated to developing the mathematical formalism. The defect is introduced as a modification of the metric from 
its otherwise Euclidean form. Beginning with a suitable choice of coordinates, the Hamiltonian of the system is constructed. The Schrödinger equation is then solved for this Hamiltonian to obtain the energy spectrum. This is followed by obtaining the various thermodynamic variables of the system using the canonical partition function. The expressions for these quantities are recast in terms of dimensionless parameters and their behavior is studied. The asymptotics are checked for consistency. In Sec. III we present the results of our study. Finally we conclude with a discussion and summary in the last section.

\section{FORMALISM}

The topological defect being introduced in the current study is a conical disclination. This entails a twodimensional (2D) conical space which is locally flat at all points except for the origin [22]. The construction of this space is to be visualized as the consequence of cutting out a sector with a certain apex angle called the deficit angle, from the ordinary 2D flat space and subsequently welding together the newly revealed edges [29]. The metric for such a space, in the usual polar coordinates $(r, \phi)$ is given by $g_{\mu \nu}=\operatorname{diag}\left(1, r^{2}\right)$. However, it has to be kept in mind that $\phi$ here has an incomplete angular range $[0,2 \pi \kappa]$ with $\kappa \neq 1$. This, being a consequence of the surgical procedure performed previously. The parameter $\kappa$ is a measure of the deficit angle. It quantifies the conicity of the surface and shall henceforth be referred to as the kink parameter. The kink here represents a singular deformity of the $2 \mathrm{D}$ conical surface at the origin. The metric described above can be expressed in terms of the complete angular coordinate $\theta$ as follows

$$
d s^{2}=\kappa^{-2} d \rho^{2}+\rho^{2} d \theta^{2} .
$$

where, $\theta$ varies in $[0,2 \pi]$. The transformation from plane polar coordinates to the new coordinate system, i.e. from $(r, \phi) \rightarrow(\rho, \theta)$ is achieved via the set of transformation equations

$$
\rho=\kappa r \quad \theta=\kappa^{-1} \phi .
$$

The curvature is measured by the quantity

$$
2 \pi \frac{\kappa-1}{\kappa} \delta^{(2)}(\rho)
$$

where $\delta^{(2)}(\rho)$ is the Dirac delta function in two dimensions [24]. Hence, for $0<\kappa<1$ we have negative curvature and for $1<\kappa<\infty$ the curvature at origin is positive. We note that the metric described here in the context of $2 \mathrm{D}$ condensed matter system also arises in the description of space-time around a cosmic string [30].

In the above described space we consider a charged spin-less quantum particle (for our purposes it has electronic mass and charge). This particle is subjected to a constant magnetic field $\mathbf{B}$ which is normal to the conical surface. The appropriate choice of magnetic vector potential that yields such a magnetic field is given in the symmetric gauge by

$$
\mathbf{A}(\rho)=\frac{B \rho}{2 \kappa} \hat{e}_{\theta}
$$

where $B=|\mathbf{B}|$. This gives rise to the standard quantized single particle Landau level states [25].

In order to model the confinement of the particle within a small region on the surface, we subject the particle to a parabolic potential of the Fock-Darwin type 27] given by

$$
V(\rho)=\frac{1}{2} M \omega_{p}^{2} \frac{\rho^{2}}{\kappa^{2}}
$$

where $M$ is the effective mass of the particle and $\omega_{p}$ is a measure of the steepness of the confinement. The appearance of the kink parameter indicates that the background space is conical. The choice of such a potential is motivated by symmetry considerations and its frequent appearance in the modeling of quantum dots with low occupancy [31].

The Hamiltonian for the particle of mass $M$, assumed to be carrying a negative charge of magnitude $e$ under minimal electromagnetic coupling, is given in the cone space coordinates $(\rho, \theta)$ as

$$
\begin{aligned}
H= & -\frac{\hbar^{2}}{2 M}\left[\frac{\kappa^{2}}{\rho} \frac{\partial}{\partial \rho}\left(\rho \frac{\partial}{\partial \rho}\right)+\frac{1}{\rho^{2}} \frac{\partial^{2}}{\partial \theta^{2}}\right]-\frac{i}{2} \frac{\hbar \omega_{c}}{\kappa^{2}} \frac{\partial}{\partial \theta} \\
& +\frac{1}{8} M \omega_{c}^{2} \frac{\rho^{2}}{\kappa^{4}}+\frac{1}{2} M \omega_{p}^{2} \frac{\rho^{2}}{\kappa^{2}}
\end{aligned}
$$

where the parameter $\omega_{c}$ introduced here is the cyclotron frequency $\omega_{c}=e B / M c$. Note the appearance of the kink parameter $\kappa \neq 1$ when one expresses the Hamiltonian in the cone space.

The general form of eigenfunctions for this Hamiltonian can be guessed from symmetry arguments. Separation of the Schrödinger equation into radial and angular components yields such a general form

$$
\psi(\rho, \theta)=\frac{1}{\sqrt{2 \pi}} e^{i m \theta} R_{n m}(\rho)
$$

The quantum numbers $n$ and $m$ are to be defined using the appropriate boundary conditions. Here, $R_{n m}(\rho)$ stands for the radial component of the wave function. The condition on $m$ is readily obtained by requiring $\psi$ to be unique under a rotation of $2 \pi$, ie $\psi(\rho, \theta)=$ $\psi(\rho, \theta+2 \pi)$. This implies that $m$ has to be an integer. The Schrödinger equation $H \psi_{n m}=E_{n m} \psi_{n m}$ yields the following equation for the radial wave function $R_{n m}(\rho)$.

$$
\begin{aligned}
& -\frac{\hbar^{2}}{2 M}\left[\frac{\kappa^{2}}{\rho} \frac{\partial}{\partial \rho}\left(\rho \frac{\partial}{\partial \rho} R_{n m}(\rho)\right)-\frac{m^{2}}{\rho^{2}} R_{n m}(\rho)\right] \\
& +\left(\frac{1}{2} \frac{\hbar \omega_{c} m}{\kappa^{2}}+\frac{1}{8} M \omega_{c}^{2} \frac{\rho^{2}}{\kappa^{4}}+\frac{1}{2} M \omega_{p}^{2} \frac{\rho^{2}}{\kappa^{2}}\right) R_{n m}(\rho) \\
& =E_{n m} R_{n m}(\rho)
\end{aligned}
$$


The procedure to solve the above equation is through a set of standard transformations, which involves the introduction of a new parameter $\Omega$ with dimension of frequency. The parameter $\Omega$ is given by

$$
\Omega=\sqrt{\omega_{p}^{2}+\left(\frac{\omega_{c}}{2 \kappa}\right)^{2}} .
$$

Following the formalism in [21] eq. (77) can be transformed to a form which permits solution in terms of the confluent-hypergeometric function (see Appendix). Our primary interest lies in the energy levels which are given by

$$
E_{n m}=\left(2 n+1+\frac{|m|}{\kappa}\right) \hbar \Omega+\frac{m \hbar \omega_{c}}{2 \kappa^{2}}
$$

If we consider the system to be at equilibrium with a heat bath at temperature $T$, the canonical partition function shall be given by

$$
\mathcal{Z}=\sum_{n, m} e^{-\beta(2 n+1) \hbar \Omega} e^{-\beta\left[\frac{|m|}{\kappa} \hbar \Omega+\frac{m \hbar \omega_{c}}{2 \kappa^{2}}\right]}
$$

where $\beta=\frac{1}{k_{B} T}$ and $k_{B}$ is the Boltzmann constant. The sum is over the discrete energy levels given in Eq. (9). Introducing dimensionless variables $\chi_{1}=\frac{\beta \hbar \Omega}{\kappa}$ and $\chi_{2}=$ $\frac{\beta \hbar \omega_{c}}{2 \kappa^{2}}$ the above expression maybe simplified to

$$
\mathcal{Z}=\frac{\sinh \chi_{1}}{4 \sinh \left(\frac{\chi_{1}+\chi_{2}}{2}\right) \sinh \left(\frac{\chi_{1}-\chi_{2}}{2}\right) \sinh \left(\kappa \chi_{1}\right)} .
$$

It is now possible to compute thermodynamic quantities from this expression of the partition function.

The internal energy $U$ for the system is given by

$$
\begin{aligned}
& U=-\frac{\partial \ln \mathcal{Z}}{\partial \beta} \\
& =-\left\{\chi_{1} \operatorname{coth}\left(\beta \chi_{1}\right)-\frac{\chi_{1}+\chi_{2}}{2} \operatorname{coth} \beta\left(\frac{\chi_{1}+\chi_{2}}{2}\right)\right. \\
& \left.-\frac{\chi_{1}-\chi_{2}}{2} \operatorname{coth} \beta\left(\frac{\chi_{1}-\chi_{2}}{2}\right)-\chi_{1} \kappa \operatorname{coth} \beta \kappa \chi_{1}\right\}
\end{aligned}
$$

Similarly one can obtain the specific heat capacity $C_{\mathrm{v}}$

$$
\begin{aligned}
& C_{\mathrm{v}}=k_{B} \beta^{2} \frac{\partial^{2} \ln \mathcal{Z}}{\partial \beta^{2}} \\
& =k_{B} \beta^{2}\left\{\frac{\left(\chi_{1}+\chi_{2}\right)^{2}}{4} \operatorname{csch}^{2} \beta \frac{\left(\chi_{1}+\chi_{2}\right)}{2}+\chi_{1}^{2} \kappa^{2} \operatorname{csch}^{2} \beta \kappa \chi_{1}\right. \\
& \left.+\frac{\left(\chi_{1}-\chi_{2}\right)^{2}}{4} \operatorname{csch}^{2} \beta \frac{\left(\chi_{1}-\chi_{2}\right)}{2}-\chi_{1}{ }^{2} \operatorname{csch}^{2} \beta \chi_{1}\right\}
\end{aligned}
$$

The Helmholtz free energy $F=-\ln \mathcal{Z} / \beta$ may be used to calculate the entropy $S$ as $S=(U-F) / T$. This yields the following expression

$$
\begin{aligned}
& S=\frac{1}{T}\left\{-\chi_{1} \operatorname{coth}\left(\beta \chi_{1}\right)+\frac{\chi_{1}+\chi_{2}}{2} \operatorname{coth}\left(\beta \frac{\chi_{1}+\chi_{2}}{2}\right)\right. \\
& \left.+\frac{\chi_{1}-\chi_{2}}{2} \operatorname{coth}\left(\beta \frac{\chi_{1}-\chi_{2}}{2}\right)+\chi_{1} \kappa \operatorname{coth}\left(\beta \kappa \chi_{1}\right)\right\} \\
& +k_{B}\left\{\ln \sinh \left(\beta \chi_{1}\right)-\ln \sinh \left(\beta \frac{\chi_{1}+\chi_{2}}{2}\right)\right. \\
& \left.-\ln \sinh \left(\beta \frac{\chi_{1}-\chi_{2}}{2}\right)-\ln \sinh \left(\beta \kappa \chi_{1}\right)-\ln 4\right\}
\end{aligned}
$$

We shall now study the variation of these quantities with the external magnetic field $B$ and temperature $T$. In order to facilitate this, it is helpful to choose certain special units which render the physical quantities $U, C_{v}$ and $S$ dimensionless. We introduce a parameter $\alpha=\omega_{c} / \omega_{p}$ to quantify the magnetic field strength in units of $M \omega_{p} c / e$ and $\xi=k_{B} T / \hbar \omega_{p}$ to represent temperature measured in units of $\hbar \omega_{p} / k_{B}$. We also introduce $\widetilde{\alpha}=\sqrt{1+\alpha^{2} / 4 \kappa^{2}}$ and $\alpha_{ \pm}=\widetilde{\alpha} \pm \alpha / 2 \kappa$.

Using these new dimensionless parameters, we have the internal energy $U$, entropy $S$ and specific heat $C_{V}$ may be expressed as

Internal energy :

$$
\begin{aligned}
& \frac{U}{\hbar \omega_{p}}=\frac{1}{\kappa}\left\{-\widetilde{\alpha} \operatorname{coth}\left(\frac{\widetilde{\alpha}}{\xi \kappa}\right)+\frac{\alpha_{+}}{2} \operatorname{coth}\left(\frac{\alpha_{+}}{2 \xi \kappa}\right)\right. \\
& \left.+\frac{\alpha_{-}}{2} \operatorname{coth}\left(\frac{\alpha_{-}}{2 \xi \kappa}\right)+\widetilde{\alpha} \kappa \operatorname{coth}\left(\frac{\widetilde{\alpha}}{\xi}\right)\right\}
\end{aligned}
$$

Specific heat :

$$
\begin{aligned}
& \frac{C_{v}}{k_{B}}=\frac{\widetilde{\alpha}^{2}}{\xi^{2}} \operatorname{cosech}^{2}\left(\frac{\widetilde{\alpha}}{\xi}\right)+\frac{\alpha_{+}{ }^{2}}{4 \kappa^{2} \xi^{2}} \operatorname{cosech}^{2}\left(\frac{\alpha_{+}}{2 \kappa \xi}\right) \\
& +\frac{\alpha_{-}{ }^{2}}{4 \kappa^{2} \xi^{2}} \operatorname{cosech}^{2}\left(\frac{\alpha_{-}}{2 \kappa \xi}\right)-\frac{\widetilde{\alpha}^{2}}{\xi^{2} \kappa^{2}} \operatorname{cosech}^{2}\left(\frac{\widetilde{\alpha}}{\kappa \xi}\right)
\end{aligned}
$$

\section{Entropy :}

$$
\begin{aligned}
& \frac{S}{k_{B}}=\frac{1}{\xi \kappa}\left\{-\widetilde{\alpha} \operatorname{coth}\left(\frac{\widetilde{\alpha}}{\xi \kappa}\right)+\frac{\alpha_{+}}{2} \operatorname{coth}\left(\frac{\alpha_{+}}{2 \xi \kappa}\right)\right. \\
& \left.+\frac{\alpha_{-}}{2} \operatorname{coth}\left(\frac{\alpha_{-}}{2 \xi \kappa}\right)+\widetilde{\alpha} \kappa \operatorname{coth}\left(\frac{\widetilde{\alpha}}{\xi}\right)\right\} \\
& +\left\{\ln \sinh \left(\frac{\widetilde{\alpha}}{\xi \kappa}\right)-\ln \sinh \left(\frac{\alpha_{+}}{2 \xi \kappa}\right)\right. \\
& \left.-\ln \sinh \left(\frac{\alpha_{-}}{2 \xi \kappa}\right)-\ln \sinh \left(\frac{\widetilde{\alpha}}{\xi}\right)-\ln 4\right\} .
\end{aligned}
$$

The asymptotic behaviour of the above expressions in the low temperature limit is instructive to look at. The internal energy $U$ in the low temperature limit is given by $U \rightarrow \hbar \Omega$, where $\Omega$ is defined earlier in eq.(8). The low temperature asymptotic form $(\xi \rightarrow 0)$ of entropy $S$ is given by

$$
\begin{aligned}
S & \approx\left(1+\frac{\alpha_{+}}{\xi \kappa}\right) e^{\frac{-\alpha_{+}}{\xi \kappa}}+\left(1+\frac{\alpha_{-}}{\xi \kappa}\right) e^{\frac{-\alpha_{-}}{\xi \kappa}} \\
& +\left(1+\frac{2 \widetilde{\alpha}}{\xi}\right) e^{\frac{-2 \widetilde{\alpha}}{\xi}}-\left(1+\frac{2 \widetilde{\alpha}}{\xi \kappa}\right) e^{\frac{-2 \widetilde{\alpha}}{\xi \kappa}}
\end{aligned}
$$


The specific heat in the low temperature limit, is approximated by the following function of temperature.

$$
\begin{aligned}
C_{v} & \approx \frac{4 \widetilde{\alpha}^{2}}{\xi^{2}} e^{\frac{-2 \widetilde{\alpha}}{\xi}}+\frac{\alpha_{+}^{2}}{\xi^{2} \kappa^{2}} e^{\frac{-\alpha_{+}}{\xi \kappa}} \\
& +\frac{\alpha_{-}^{2}}{\xi^{2} \kappa^{2}} e^{\frac{-\alpha_{-}}{\xi \kappa}}-\frac{4 \widetilde{\alpha}^{2}}{\xi^{2} \kappa^{2}} e^{\frac{-2 \widetilde{\alpha}}{\xi \kappa}}
\end{aligned}
$$

\section{RESULTS AND DISCUSSION}

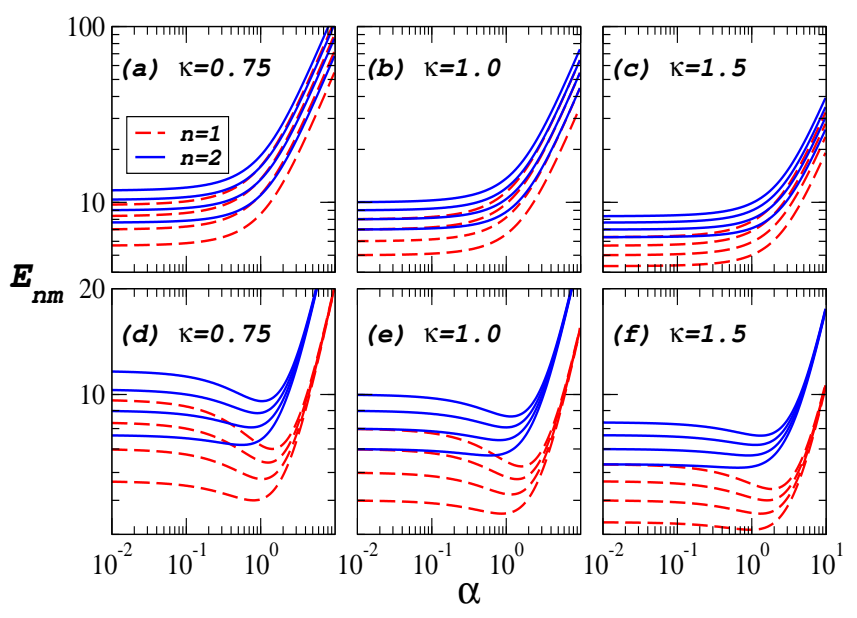

FIG. 1. (Color online) The low lying energies of the LandauFock-Darwin energy spectrum for various values of the kink parameter $\kappa$. The upper panel $(\mathrm{a}-\mathrm{c})$ shows the spectra for positive values of the quantum number $m=2,3,4,5$ (lower to the upper) and the lower panel (d-f) corresponds to negative values of $m=-2,-3,-4,-5$ with decreasing magnitude $|m|$ from upper to the lower curves.

\section{A. The Energy Spectrum}

The Landau-Fock-Darwin energy spectrum is given by Eq. (9). Fig. (11) shows the variation of $E_{n m}$ with the external magnetic field parameter $\alpha$, for a few chosen values of the kink parameter $\kappa=0.75,1.0,1.5$. The behaviour of the energy levels is different for positive and negative values of the quantum number $m$. The figure shows the variation of $E_{n m}$ with $\alpha$ for $n=1,2$. In the upper panel we show the case when the integer $m$ is assumed to take positive values $2,3,4$ and 5 for each $n$. The behaviour at very low magnetic field shows that $E_{n m}$ is independent of $\alpha$ for $\alpha \lesssim 10^{-2}$. In this low magnetic field regime one finds the usual degeneracies of $(n, m)$ pairs since $E_{n m} \approx(2 n+1+|m| / \kappa) \hbar \omega_{p}$. In our case with $\kappa=1.0$ this occurs, for example between $(n, m)$ pairs like $[(2,2),(1,4)],[(2,3),(1,5)]$ and $[(2,4),(1,6)]$. These degeneracies starts to get lifted when the external magnetic field is sufficiently high $(\alpha \approx 1)$. At very high magnetic fields $\left(\omega_{c}>>\omega_{p}\right)$ and for $m>0$, we have
$E \rightarrow\left[(2 n+1) / 2 \kappa+m / \kappa^{2}\right] \hbar \omega_{c}$ leading to new degeneracies. In the relatively high magnetic field region of $\alpha \approx 10$ one can readily observe that curves for all $(n, m)$ are monotonically increasing with nearly fixed slopes. The transition between these extreme behaviours occurs in the intermediate field region of $\alpha \approx 1$. We note, that in the intermediate and large magnetic field regions the difference between the energy levels with the same value of $n$ but different values of $m$ is larger as compared to the low field region. This is owing to the fact that $\omega_{c}$ is larger for higher magnetic fields. For example the level corresponding to $(1,3)$ is higher than $(2,2)$. The energy levels shift in magnitude for changing $\kappa$ which implicitly affects the degeneracy pattern.

The figures in the lower panel 1(d)-1(f) shows the spectrum for negative $m$ values. The low magnetic field behaviour is the same as for the positive $m$ case. However at large magnetic fields the term $m \hbar \omega_{c} / 2 \kappa^{2}$ starts to play an important role and cancels the term $|m| \hbar \Omega / 2 \kappa$ in this regime. The spectrum becomes independent of $m$ and only depends on $n$. The increase of $E_{n m}$ is approximately linear with magnetic field $\alpha$. The transition between the two regimes occurs again at $\alpha \approx 1$.

Figure 2 shows the variation of energy with the kink parameter $\kappa$ for three different values of the applied magnetic field. Fig 2(a), (b) and (c) show the variation for negative $m$ values $(m=-1,-3,-5)$ corresponding to $n=1,2$. The curves show a monotonic decrease of $E_{n m}$ with $\kappa$ in all the three regimes of magnetic field $\alpha$. The value $\kappa=1$ corresponds to the case with no topological defect. We note an asymmetry in the nature of variation of $E_{n m}$ about this value of $\kappa$. The energy levels are a decreasing function of $\kappa$ for both $\kappa \geq 1$ and $\kappa<1$ showing that positive and negative deficit angles point towards fundamentally different physical situations. The

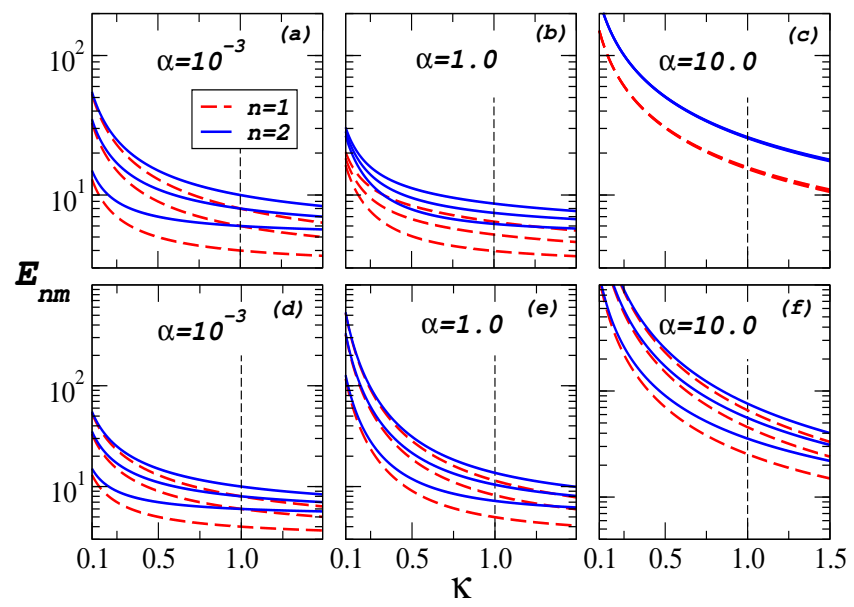

FIG. 2. (Color online) The first few levels of the LandauFock-Darwin energy spectrum as a function of the kink parameter $\kappa$. The upper panel corresponds to negative values of the quantum number $m=-1,-3,-5$. The lower panel shows the same for positive values of $m=1,3,5$. Three magnetic field values are chosen with $\alpha=10^{-3}, 1.0,10.0$. 
expression for $E_{n m}$ diverges as $\kappa \rightarrow 0$. This, however is of no real consequence since $\kappa=0$ corresponds to an unphysical divergent curvature at the origin.

The vertical dotted line indicating the case without any defect $(\kappa=1)$ passes through the point of intersection of the energy levels. These points correspond to the degenerate energy levels at low magnetic field. The degeneracy of the $(n, m)$ levels for $\kappa=1$ are seen to get lifted for $\kappa \neq 1$ as the energy levels for different $m$ vary differently with $\kappa$. In Fig. 2(c) the different $m$ levels for a given $n$ are degenerate and remain so, irrespective of $\kappa$. The figures 2(d)-2(f) show a similar variation for positive $m$ values. Whereas the degeneracies at weak magnetic field ( Fig. 2(d)) gets lifted for $\kappa \neq 1$ there are new degeneracies that are created at higher magnetic fields. This is seen in Fig. 2(e)-2(f) where non-degenerate energy levels at $\kappa=1$ intersect each other at $\kappa \neq 1$ showing the emergence of accidental degeneracies that did not exist in the defect free theory.

\section{B. Thermodynamic properties}

The non-interacting spinless charged particles are assumed to be in equilibrium with a heat reservoir at temperature $T$. The starting point of the thermodynamic analysis is the evaluation of the partition function for the energy spectra given in Eq. (9). The Landau-Fock-
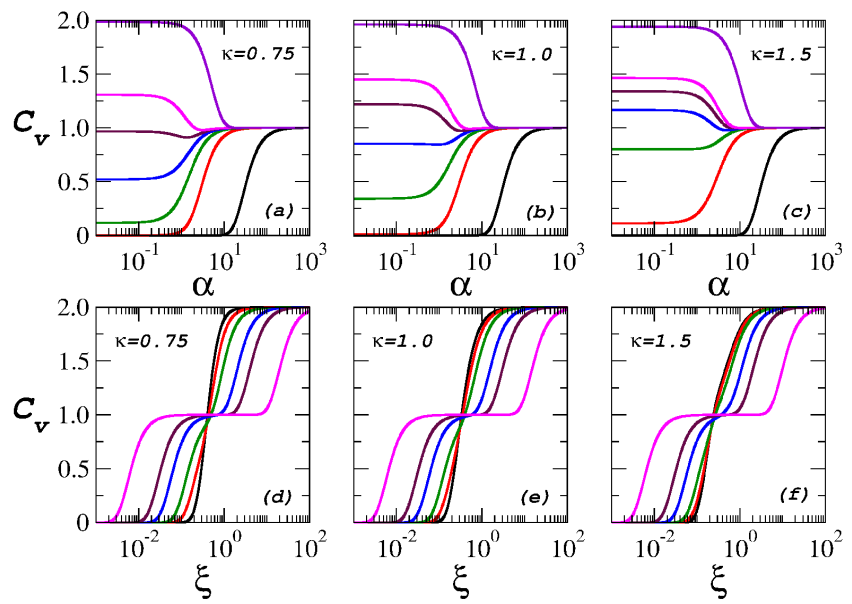

FIG. 3. (Color online) The $C_{\mathrm{v}}$ shown here is in units of $k_{B}$. The upper panel shows the variation of $C_{\mathrm{v}}$ with magnetic field for various values of temperature $\xi=$ $0.01,0.1,0.2,0.3,0.4,0.5,2.0$ (curves from lower to upper). At high magnetic fields $C_{\mathrm{v}}$ attains the value 1.0 as the spectrum reduces to the free Landau levels with no confinement. The high temperature value of $C_{\mathrm{v}}$ for moderate to low magnetic fields is 2 as the confinement term dominates at these regimes. The Lower panel shows the variation of $C_{\mathrm{v}}$ with temperature for $\alpha=0.001,1.0,2.0,5.0,10.0,50.0$ (left to right in the upper right corner of the figures). Here again the plateau in $C_{\mathrm{v}}$ is seen for the high magnetic fields and only at high temperatures $C_{\mathrm{v}}$ attains the value 2.0 .
Darwin Hamiltonian has two energy scales associated with the two frequencies $\omega_{p}$ (which fixes the strength of the parabolic confinement) and $\omega_{c}$, the cyclotron frequency related to the external magnetic field. The relative strengths of these frequencies are expected to govern the equilibrium behaviour of the system. The thermodynamic properties of interest, depend on the temperature $\xi$ and external magnetic field $\alpha$, expressed in our chosen convenient energy unit $\hbar \omega_{p}$. The parameters in the Hamiltonian $\left(\omega_{p}, \omega_{c}, \kappa\right)$ have a crucial interplay in determining the responses of the system. The $\kappa=1$ case with no defects has been studied in earlier works [15, 26]. It is important to note that for $\kappa=1$, the limiting behaviour of the system for $\omega_{p} \rightarrow 0$ (or equivalently $\left.\omega_{c}>\omega_{p}\right)$ and $\omega_{c} \rightarrow 0$ are entirely different and describe two completely distinct physical situations. The former describes a pure Landau problem of a free particle without any confinement, whereas the latter describes a particle in a two dimensional parabolic well without a coupling to an external magnetic field. The $\omega_{p} \rightarrow 0$ limit has a pure quantum mechanical Landau-level spectra of a one-dimensional oscillator and has the degeneracy that depends on the size of the system. The energy spectra for the case $\omega_{c} \rightarrow 0$ mimics that of a $2 \mathrm{D}$ oscillator. The translational symmetry of the pure Landau level situation is lost completely in the other extreme limit of a pure confinement problem. The general Landau-Fock-Darwin solution interpolates between these extreme cases. In the presence of $\kappa \neq 1$ the same qualitative features are expected. However, the role of $\kappa$ needs to be explored and is subsequently discussed in this paper.

We follow the Gibbs formalism to compute thermodynamic quantities like free energy, entropy and specific heat. In this approach, the thermodynamic response functions are obtained as derivatives of the partition function. The canonical partition function (see Eq. 10) is obtained for the Hamiltonian in Eq. (5). In the final form, this partition function (see Eq. (11)) is seen to diverge in the limit $\omega_{c}>>\omega_{p}$ (or $\alpha>>1$ ) since, $\chi_{1}$ and $\chi_{2}$ are equal in this limit. This singularity of the partition function, when the confinement strength is vanishingly small, has been addressed in 26] and maybe regularized by putting certain cutoffs to the smallest value that $\omega_{p}$ can take. This cutoff depends on the temperature and the degeneracy of the pure Landau level. The thermodynamic quantities like $F, U, S$ and $C_{\mathrm{v}}$ however, manifest no such singularity.

Figure 3 shows the variation of $C_{\mathrm{v}}$ with magnetic field $\alpha$ and temperature $\xi$ for different values of the kink parameter $\kappa$. The variation of $C_{\mathrm{v}}$ with $\alpha$ shows that for weak external magnetic field and low temperatures $C$ asymptotically approaches zero. However, in this weak $\alpha$ regime, at high temperatures $C_{v} \rightarrow 2 k_{B}$ asymptotically. This is in consonance with the equipartition principle. The low $\alpha$ end behaves like a 2D oscillator (hence the factor 2). In the high magnetic field regime ( $\alpha$ large), $C_{\mathrm{v}}$ saturates to $k_{B}$. This region corresponds to the pure Landau level with the energy spectrum of an 1D oscil- 

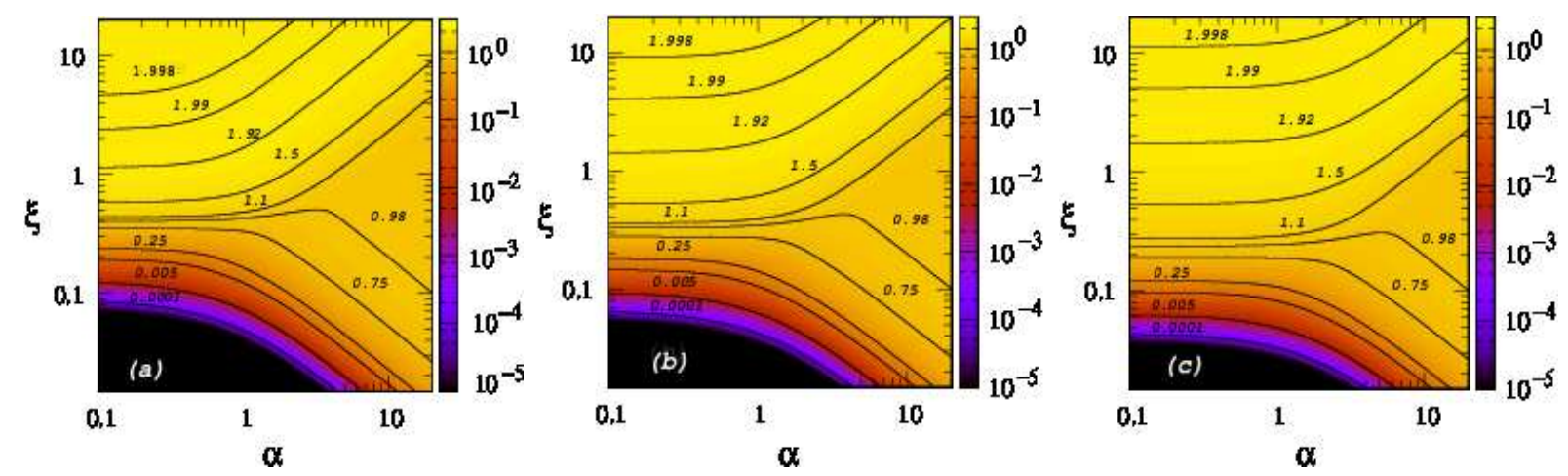

FIG. 4. (Color online) The contour map for specific heat $c_{\mathrm{v}}$ in the $(\xi, \alpha)$ phase plane, for three values of the kink parameter (a) $\kappa=0.75$, (b) $\kappa=1.0$, (c) $\kappa=1.5$.

lator. The qualitative features are similar when $\kappa \neq 1$. However, we see that changing $\kappa$ from 0.75 to 1.5 continuously, leads to a shift of the curves from the lower end towards the upper. This can be qualitatively ascribed to the fact that $\kappa$ appears as a multiplicative factor to $\xi$ in the expression for $C_{\mathrm{v}}$ and a change of $\kappa$ roughly amounts to a recalibration of the temperature scale.

The variation of $C_{\mathrm{v}}$ with temperature $\xi$ is shown in the lower panel of Fig. 3. When the value of $\alpha$ is small, the rise of $C_{\mathrm{v}}$ with temperature is steep, and in a very small temperature range, $C_{\mathrm{v}}$ rises from zero to a stable value of $2 k_{B}$. Beyond the transition temperature, $C_{\mathrm{v}}$ remains flat at this value. In this situation the system is essentially dominated by the parabolic confining potential and the physics of the Landau levels is missing. The situation is considerably different when $\alpha$ is large. Here the effect of confinement is weak and $C_{\mathrm{v}}$ attains a plateau like level when temperature is increased. The value of $C_{v}$ remains constant at $k_{B}$ for a range of temperatures after which it rises to $2 k_{B}$ only at high values of $\xi$. The formation

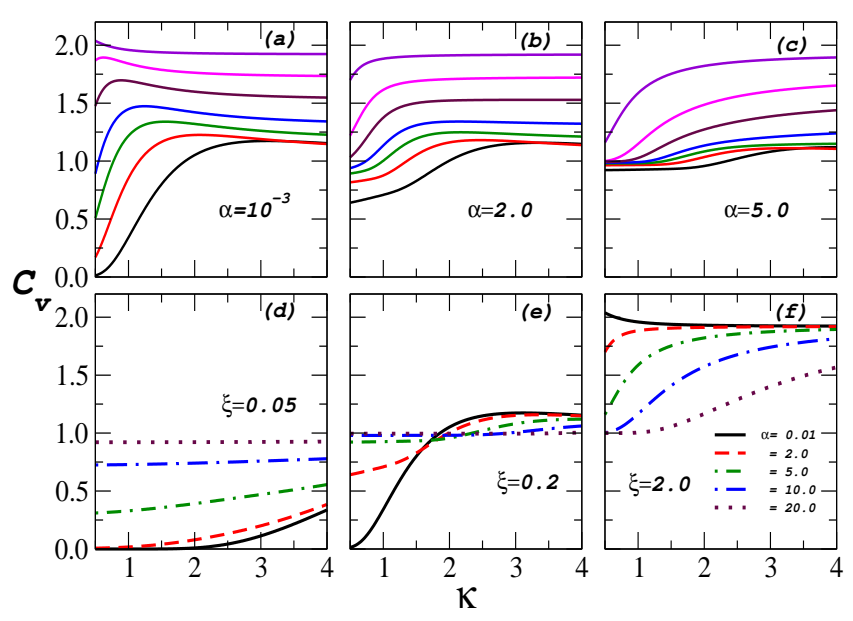

FIG. 5. (Color online) The dependence of $C_{\mathrm{v}}$ on the kink parameter $\kappa$ for different values of external magnetic field $\alpha$ and temperature $\xi$. For the upper panel $\xi=$ $2.0,1.0,0.7,0.5,0.4,0.3,0.2$ (top to bottom). of the plateau can be ascribed to the dominance of the Landau 1D oscillator spectrum at high magnetic fields as opposed to the 2D oscillator spectrum of the parabolic well when the magnetic field is weak. The extent of the plateau region is found to be sensitive to $\kappa$. We shall discuss this $\kappa$ dependence later.

Figure 4 shows the contour map of $C_{\mathrm{v}}$ in the $(\alpha, \xi)$ plane. At very low temperatures, $C_{\mathrm{v}} \rightarrow 0$ except, when the external magnetic field is large. The lower left corner of the $(\alpha, \xi)$ plane corresponds to this phase where $C_{\mathrm{v}}$ is small. Increasing the temperature at small values of $\alpha$ leads to a monotonic increase of $C_{\mathrm{v}}$ to its saturated value of $2 k_{B}$ (upper left corner of the phase diagram). At such low values of $\alpha$ there is hardly any Landau coupling to the magnetic field. The Landau plateau occurs at large $\alpha$ when the energy spectrum approaches the Landau levels. This is the forked region of the contour map, where, for a considerable range of intermediate temperatures the value of $C_{\mathrm{v}}$ remains at the $k_{B}$ level, and only increases to $2 k_{B}$ at still higher temperatures (this is not seen in the phase diagram and occurs for values of $\xi$ even above the upper right corner). The extent of the forking region (plateau in $C_{\mathrm{v}}$ depends on the kink parameter. Infact, it is seen to decrease with increasing $\kappa$. This can be understood by noting that a changing $\kappa$ can be equivalently seen as changing $\xi$ with a fixed $\kappa$. The qualitative features of the phase diagram remain the same when $\kappa$ is varied. However, there are quantitative changes which we shall discuss now.

Figure 5 shows the variation of $C_{\mathrm{v}}$ with $\kappa$. At high temperatures, $C_{\mathrm{v}}$ is not sensitive to $\kappa$ unless the magnetic field $\alpha$ is also very high. This is seen in the figures $5(\mathrm{a})-(\mathrm{c})$. The specific heat is however very sensitive to $\kappa$ at low temperatures. Increasing $\kappa$ can be equivalently interpreted as a scaling of $\alpha$ and this explains the plateau (characteristic of large $\alpha$ ) when $\kappa$ is large. At large $\alpha$ (figure $5(\mathrm{c})$ ), all the low temperature curves cluster around the $k_{B}$ level and stabilizes at the $2 k_{B}$ level only for high temperatures. Figure 5(e) shows that there is a cross over of $C_{\mathrm{v}}$ at a certain value of $\kappa$. This implies that at some intermediate low temperatures $C_{\mathrm{v}}$ is not much sensitive to the changes in the magnetic field for certain values 
of $\kappa$. At higher temperatures, however, $C_{\mathrm{v}}$ saturates to $2 k_{B}$. This growth is slower for the curves corresponding to large $\alpha$ values which tends to stay in the plateau region as compared to the case when $\alpha$ is small. Here, we see that $\kappa$ essentially re-calibrates the temperature scale.

Figure 6 shows the behaviour of the entropy as a function of magnetic field and temperature. The competition between the variables $\xi$ and $\alpha$ decides the degree of order in the system. We find that the asymptotic form of $S$ in Eq. (18) is valid for a certain value of $\xi$ that depends on the magnetic field and $\kappa$. This region of validity of this limiting form of entropy is shown by broken lines in Fig. 6 (a)-(c). The third law of thermodynamics is respected and we have $S \rightarrow 0$ as $\xi \rightarrow 0$. The growth of entropy from the low temperature ordered regime to the disordered state at high temperature, depends on the magnetic field. The growth is steeper for higher magnetic fields. However at very high temperatures the magnetic field dependence keeps decreasing. Figures 6 (d-f) shows the variation of entropy with magnetic field. At very high magnetic field there is a slowing down on the rate at which $S$ increases. This feature is seen for a wide range of temperatures. The effect of $\kappa$ here is clearly that of a scaling parameter that re-calibrates the temperature scale $\xi$.

\section{SUMMARY AND CONCLUSION}

In this work, we have carried out a study of the thermodynamic ramifications of a conical defect, in the context of Landau-Fock-Darwin problem. The competing behaviour of the temperature and magnetic field is noted, and how a change in the kink parameter influences this. The variation of quantities like specific heat and entropy
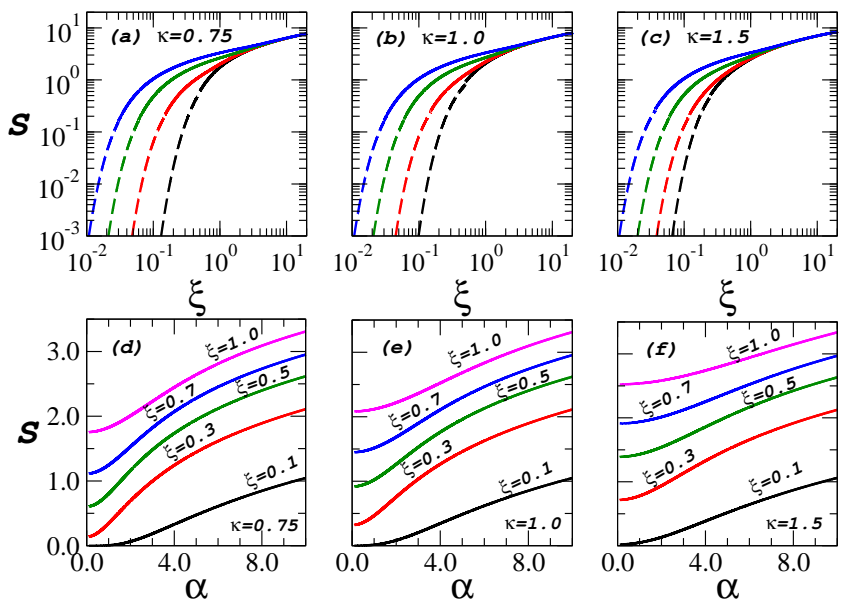

FIG. 6. (Color online) The upper panel (a-c) shows the variation of $S$ with $\xi$ for various values of $\alpha$ and $\kappa$ with $\alpha=10.0,5.0,2.0,0.1$ (from left to right). The broken lines indicate the temperature range for validity of the low temperature asymptotic behaviour of $S$. The lower panel shows the $\alpha$ dependence of entropy for specific temperatures and $\kappa$.

with the kink parameter illustrates the physical effect of the disclination to be a sort of recalibration of the temperature scale. Also of note are the essential non trivialities inherent in the Landau-Fock-Darwin problem with respect to the symmetry of the system. These are recovered here in the presence of the conical defect as is illustrated by the step in the specific heat curve at high magnetic fields, which reflects the interpolation of the behaviour between a 1D and a 2D oscillator.

We conclude by noting that it is possible to extend this analysis to further studies which could incorporate discrete lattice structure and interactions in the presence of this class of topological defects.
[1] T. Ando, A. B. Fowler, and F. Stern, Rev. Mod. Phys. 54, 437 (1982)

[2] S. M. Reimann and M. Manninen Rev. Mod. Phys. 74, 1283 (2002)

[3] R. C. Ashoori, Nature 379, 413 (1996)

[4] M. A. Reed, J. N. Randall, R. J. Aggarwal, R. J. Matyi, T. M. Moore, and A. E. Wetsel, Phys. Rev. Lett. 60, 535 (1988)

[5] J. Cibert, P. M. Petroff; G. J. Dolan, S. J. Pearton, A. C. Gossard, and J. H. English, Appl. Phys. Lett. 49, 1275 (1986)

[6] Ch. Sikorski and U. Merkt. Phys. Rev. Lett. 62, 2164 (1989)

[7] T. Chakraborty, Quantum Dots: A Survey of the Properties of Artificial Atom (Elsevier Science, Amsterdam, 1999)

[8] K. Kash, A. Scherer, J. M. Worlock, H. G. Craighead, and M. C. Tamargo, Appl. Phys. Lett. 49, 1043 (1986)

[9] H. Temkin, G. J. Dolan, M. B. Panish, and S. N. G. Chu, Appl. Phys. Lett. 50, 413 (1987)

[10] O. Makarovsky, O. Thomas, A. G. Balanov, L. Eaves, A.
Patan, R. P. Campion, C. T. Foxon, E. E. Vdovin, D. K. Maude, G. Kiesslich, and R. J. Airey, Phys. Rev. Lett. 101, 226807 (2008)

[11] T. P. Smith, III, K. Y. Lee, C. M. Knoedler, J. M. Hong,and D. P. Kern, Phys. Rev. B 38, 2172 (1988).

[12] R. C. Ashoori, H. L. Stormer, J. S. Weiner, L. N. Pfeiffer, S. J. Pearton, K. W. Baldwin, and K. W. West Phys. Rev. Lett. 68, 3088 (1992); R. C. Ashoori, H. L. Stormer, J. S. Weiner, L. N. Pfeiffer, K. W. Baldwin, and K. W. West, Phys. Rev. Lett. 71, 613 (1993)

[13] W. Hansen, T. P. Smith, III, K. Y. Lee, J. A. Brum, C. M. Knoedler, J. M. Hong, and D. P. Kern, Phys. Rev. Lett. 62, 2168 (1989)

[14] T. Demel, D.'Heitmann, P. Grambow, and K. Ploog, Phys. Rev. Lett. 64, 788 (1990)

[15] B. Boyacioglu and A. Chatterjee. J. Appl. Phys. 112, 083514 (2012)

[16] P. A. Maksym and T. Chakraborty. Phys. Rev. Lett.65, 108 (1990)

[17] N. D. Mermin, Rev. Mod. Phys. 51, 591 (1979)

[18] E. Kröner, in Physics of Defects, R. Balian et al. (North- 
Holland, Amsterdam, 1981)

[19] M. Kléman, Points, Lines and Walls(Wiley, New York,1983)

[20] V. A. Osipov, Phys Lett. A 164, 327, (1992)

[21] C. Furtado, B. G. C. da Cunha, F. Moraes, E. R. Bezerra de Mello, V. B. Bezzerra, Phys. Lett. A 195, 90 (1994)

[22] E. R. Bezerra de Mello and V. B. Bezerra, J. Math. Phys. 36, 5297 (1995)

[23] C. Furtado and F. Moraes, Europhys. Lett. 45, 279 (1999)

[24] G. de A. Marques et al, J. Phys. A 34, 5945 (2001)

[25] L. D Landau. Z. Phys. 45, 430 (1927); reprinted in the "Collected papers of L. D. Landau" ed C. Ter Haar. Pergamon Press, Oxford.

[26] J. Kumar, P. A. Sreeram, and S. Dattagupta, Phys. Rev. E, 79, 021130 (2009); J. Kumar and E. Kamil, arXiv:1010.1205v4; S. Dattagupta, J. Kumar, S. Sinha, and P. A. Sreeram, Phy. Rev. E 81, 031136 (2010)

[27] C. G. Darwin. Proc. Camb. Philos. Soc. 27, 86 (1930)

[28] D. Abergel, V. Apalkov, J. Berashevich, K. Ziegler, and T. Chakraborty, Adv. Phys. 59, 261 (2010); A. H. Castro Neto et al, Rev. Mod. Phys. 81, 109 (2009)

[29] V. Volterra, Ann. Éc. Norm. Sup. 24, 401 (1907)

[30] A. Vilenkin, Phys. Rev. D 23, 852 (1981)

[31] A. Kumar, S. E. Laux, F. Stern, Phys. Rev. B 42, 5166 (1990)

\section{Appendix}

To solve the radial eigenvalue equation (7), we introduce $\zeta=\rho^{2} M \Omega / \hbar$. This transformation yields the following equation

$$
\zeta \frac{\partial^{2} R(\zeta)}{\partial \zeta^{2}}+\frac{\partial R(\zeta)}{\partial \zeta}+\Xi(\zeta) R(\zeta)=0
$$

where we have used

$\Xi(\zeta)=\frac{\beta}{\kappa^{2}}-\frac{\zeta}{4 \kappa^{4}}-\frac{m^{2}}{4 \kappa^{2} \zeta} \quad$ and $\quad \beta=\frac{1}{2}\left(\frac{E_{m n}}{\hbar \Omega}-\frac{\omega_{c} m}{2 \kappa^{2} \Omega}\right)$.

Using variables $\zeta^{\prime}=\zeta / \kappa^{2}$ and $m^{\prime}=m / \kappa$ we have

$$
\zeta^{\prime} \frac{\partial^{2} R\left(\zeta^{\prime}\right)}{\partial \zeta^{\prime 2}}+\frac{\partial R\left(\zeta^{\prime}\right)}{\partial \zeta^{\prime}}+\Xi^{\prime}\left(\zeta^{\prime}\right) R\left(\zeta^{\prime}\right)=0
$$

where the new function $\Xi^{\prime}$ is

$\Xi^{\prime}\left(\zeta^{\prime}\right)=\beta^{\prime}-\frac{\zeta^{\prime}}{4}-\frac{m^{\prime 2}}{4 \zeta^{\prime}}, \quad$ with $\quad \beta^{\prime}=\frac{1}{2}\left(\frac{E_{m n}}{\hbar \Omega}-\frac{\omega_{c} m^{\prime}}{2 \kappa \Omega}\right)$.
Assuming $R\left(\zeta^{\prime}\right)$ to be of the form

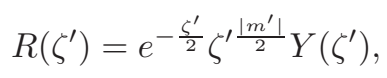

the equation (A.2) reduces to

$$
\begin{aligned}
\zeta^{\prime} \frac{\partial^{2} Y}{\partial \zeta^{\prime 2}} & +\left(\left|m^{\prime}\right|+1-\zeta^{\prime}\right) \frac{\partial Y}{\partial \zeta^{\prime}} \\
& +\left(\beta^{\prime}-\frac{\left|m^{\prime}\right|}{2}-\frac{1}{2}\right) Y=0 .
\end{aligned}
$$

The solution to this equation is given in terms of the confluent-hypergeometric function as

$$
Y\left(\zeta^{\prime}\right)=F\left[-\left(\beta^{\prime}-\frac{\left|m^{\prime}\right|}{2}-\frac{1}{2}\right),\left|m^{\prime}\right|+1 ; \zeta^{\prime}\right]
$$

The requirement of boundedness of $R\left(\zeta^{\prime}\right)$ as $\zeta^{\prime} \rightarrow \infty$ is met if

$$
\beta^{\prime}-\frac{\left|m^{\prime}\right|}{2}-\frac{1}{2}=n,
$$

where $n$ is a non-negative integer. From this boundary condition (after substituting $m / \kappa$ in place of $m^{\prime}$ ) the eigenenergies are given by

$$
E_{n m}=\left(2 n+1+\frac{|m|}{\kappa}\right) \hbar \Omega+\frac{m \hbar \omega_{c}}{2 \kappa^{2}}
$$

The eigenfunctions corresponding to these eigenvalues are obtained after imposing the requirement that for integral values of $n$, the confluent hypergeometric function reduces to Laguerre polynomials given as

$$
L_{n}^{\alpha}\left(\zeta^{\prime}\right)=\frac{\Gamma(\alpha+n+1)}{\Gamma(\alpha+1) n !} F\left(-n, \alpha+1 ; \zeta^{\prime}\right)
$$

here $\Gamma(n)=(n-1)$ ! is the usual gamma function. Thus the eigenfunctions are of the form

$$
R(\zeta)=C e^{-\frac{\zeta}{2 \kappa^{2}}}\left(\frac{\zeta}{\kappa^{2}}\right)^{\frac{|m|}{2 \kappa}} L_{n^{\kappa}}^{\frac{|m|}{\kappa}}(\zeta)
$$

where $C$ is the constant of normalization. The first term in the product represents a Gaussian in the variable $\rho$ whose spread is now determined by the degree of disclination. The localization of the wave function is hence sensitive to $\kappa$ and consequently all probability densities are affected by the degree of conicity. The appearance of $|m| / \kappa$ indicates the deficit/surplus of the polar angle quantified through $\kappa$. 